\title{
Real-Time Face Detection and Recognition on LEGO Mindstorms NXT Robot*
}

\author{
Tae-Hoon Lee \\ Center for Cognitive Robotics, Korea Institute of Science and Technology, \\ 39-1 Hawolgok-dong, Seongbuk-gu, Seoul 136-791, Republic of Korea
}

\begin{abstract}
This paper addresses a real-time implementation of face recognition system with LEGO Mindstorms NXT robot and wireless camera. This system is organized to capture an image sequence, find the features of face in the images, and recognize and verify a person. Moreover, this system can collect the facial images of various poses due to movable robot, which enables this system to increase performance. The current implementation uses the LDA(Linear Discriminant Analysis) learning algorithm considering the number of training data. We have made several tests on video data, and measured the performance and the speed of the proposed system in real environment. Finally, the result has confirmed that the proposed system is better than conventional PC-based systems.
\end{abstract}

Keywords: LEGO Mindstorms NXT robot, real-time face recognition.

\section{Introduction}

During the past few years, we have witnessed the explosion in interest and progress in automatic face recognition technology. Face recognition technologies have been developed and come into our life. For instance, applications such as intelligent building, PC security system based on face analysis start to appear in recent years.

For applying face recognition technologies to real applications, many kinds of systems have been developed before 21 century. In the early 1990s, Gilbert et al. introduced a real-time face recognition system using custom VLSI hardware for fast correlation in an IBM compatible PC 2]. Five years later, Yang et al. introduced a parallel implementation of face detection algorithm using a TMS320C40 chip 3. On the other hand, IBM introduced a commercial chip, ZISC which can compute the classification in RBF(Radial Basis Function) based neural network 4].

However, these efforts did not make successful results because they could not cope with real problems caused by illumination change, pose variation, human aging, lens distortion, and so forth. So recent face researches are focused on solving these problems. For illumination invariant face recognition, S. Zhou and

\footnotetext{
* This research was performed when the author was a summer student intern at the Center for Cognitive Robotics, Korea Institute of Science and Technology.
} 
R. Chellappa proposed rank constraint recognition [5] and A. Georghiades et al. proposed 'Illumination cone'. For pose variation, R. Gross proposed 'Eigen lightfields' [7] and 3D face recognition based on morphable model was proposed by V. Blanz and T. Vetter [8].

These approaches deal with illumination changes using multiple training data from database, which have already collected in well-structured studio. However, it can be difficult to collect these facial data in real environment. The limited number of registered facial images is also one of the reason why the recognition rate decreases. Since face recognition system has generally a fixed position, it requires well-posed and frontal faces. Recently for overcoming this limitation, many researches have been reported 1$]$.

In order to solve training data problem, we adopt movable camera using wireless camera and collect more training data instead of improving learning algorithms. We can collect multiple facial data in different poses and conditions using active robot, which is controlled by predefined rules. Then LDA can categorize these data into each manifold.

The remaining parts of this paper are organized as follows: In Section 2, we introduce the hardware structure regarding the movable camera. Our face recognition processes by the LDA learning are presented in Section 3. In Section 4 , the applicability of the proposed method is illustrated via some experiments. Finally, in Section 5, concluding remarks are given.

\section{Hardware Design}

Most of face recognition system is running using non-movable camera. Therefore it has a defect that can not deal with various face pose. In order to cope

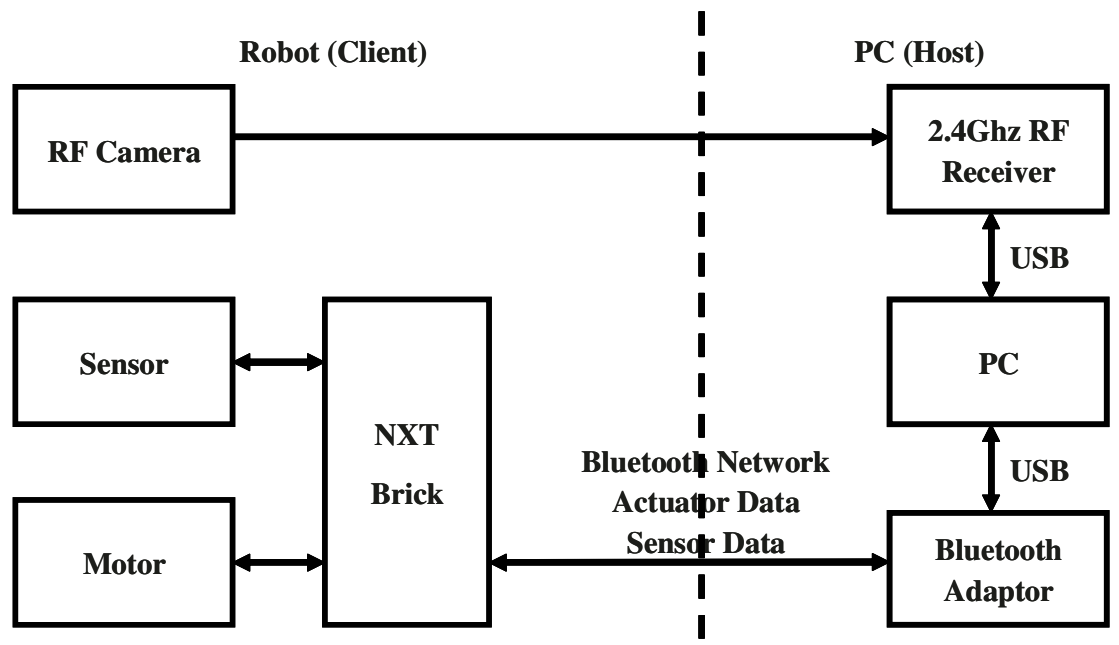

Fig. 1. The block diagram of the proposed system 
with diverse facial images, the face recognition system has to train many faces collected from well-designed studio or recognize a well-posed frontal face. Considering these restricted conditions, we construct a prototype system based on LEGO Mindstorms NXT robot with wireless camera. The LEGO Mindstorms NXT is well known as a tiny embedded system for students 9 . This system can collect arbitrarily posed facial images and train them.

In this section, we introduce our system's hardware architecture. This system is composed of server and client system as shown in Fig. 1. The former is exactly same as the conventional $\mathrm{PC}$, which plays a role on face recognition process and the latter consists of a camera and a LEGO Mindstorms NXT robot. Two systems can communicate with each other via Bluetooth.

The LEGO Mindstorms NXT brick has a 32-bit Atmel ARM7 processor with Atmel AVR coprocessor. Each processor has 256KB Flash memory and 512 byte memory respectively. Additionally it has Bluetooth module for wireless communication, which a server can control its motor module. The LEGO Mindstorms NXT robot has too small memory to recognize a person(See Table 1 So we attached a wireless camera and make communication between the server and the camera using radio frequency $(\mathrm{RF})$ devices. Host system analyzes the video frames and sends the control signal to LEGO Mindstorms NXT robot for collecting data while moving around. The LEGO Mindstorms NXT robot is decorated with LEGO blocks and camera, which is shown in Fig. 2.

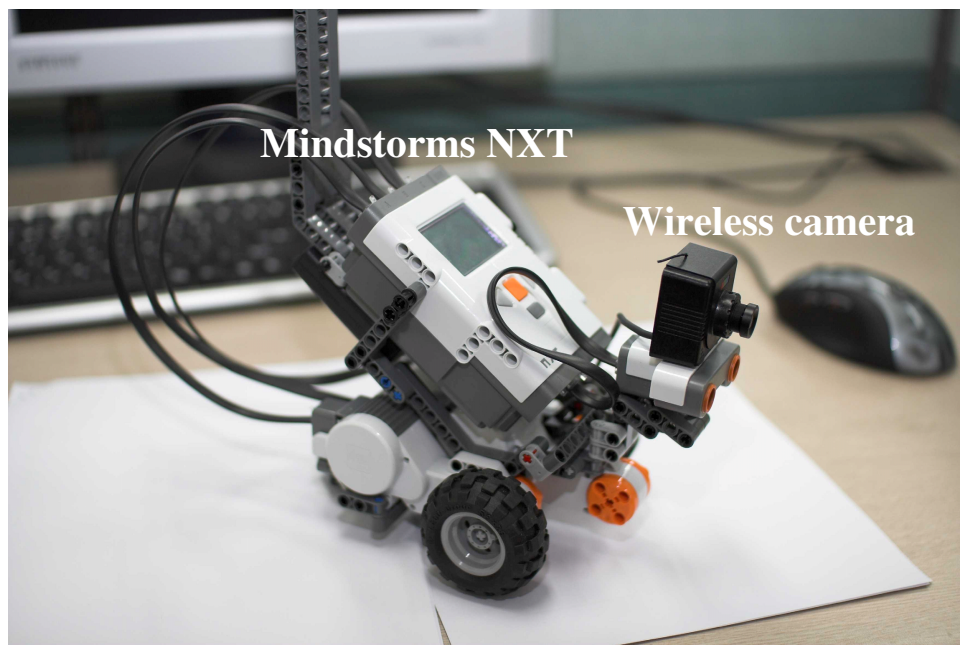

Fig. 2. LEGO Mindstorms NXT robot with wireless camera

So we can take a randomly posed facial image by camera with mobile robot. Our mobile robot is made up of the LEGO Mindstorms NXT robot. A robot's controller is NXT Brick that is small embedded system that play a role in the brain of LEGO Mindstorms NXT robot. Specification of this hardware is shown as Table 115. 
Table 1. Specification of LEGO Mindstorms NXT

\begin{tabular}{|c|c|}
\hline Processor & 32-bit ARM7 250MHz \\
\hline RAM & 64 Kilo Byte \\
\hline Storage & 256 KB Flash Memory \\
\hline Controller & Atmel AVR microcontroller $4 \mathrm{MHz}$ \\
\hline RAM & 512 Byte \\
\hline Display & 100x64 pixel LCD matrix \\
\hline Input & four 6 -wire cable digital platform \\
\hline Output & three 6-wire cable digital platform \\
\hline
\end{tabular}

As mentioned, face recognition process requires high performance computing power. However, NXT brick has low computing power. Since we can not process image information on the NXT Brick, we implemented host PC-based face recognition system and construct wireless communication between robot and PC. On this system, the wireless camera captures video frames and send them to host PC. Robot works as actuator of camera by the signal from host PC. In other words, we take pictures using the wireless camera and transfer image data through radio frequency using transmitter. On the $\mathrm{PC}$, our program receives an image from USB RF Receiver, processing image data and controlling robot using Bluetooth and RF communications.

\section{Face Analysis}

\subsection{Face Detection and Feature Extraction}

As the first step of the entire process, face detection affects greatly the overall system performance. In fact, a successful face detection is prerequisite to the success of following face recognition and verification tasks. Our system extracts faces using OpenCV face detector [14]. OpenCV face detector uses Haar cascade classifier. This algorithm was proposed by P. Viola and M. Jones [13]. Basically this approach is based on Adaboost, which is known as one of the best performance and quality methods. We have trained much enough faces with various poses that is also collected by movable robots. After face detection, we adjust the size of facial image into default rectangle, and extract the face region with ellipse mask because face image contains background area. And then we apply the histogram equalization processing. It is known that the histogram equalization is effective under quite a different illumination condition. Even if it could not normalize locally illuminated shape, it works in general illumination changes by windows and lights in indoor environment.

\subsection{Face Classification}

The classification of feature vectors in face images needs a essential compression of large images into a small vector. We used PCA(Principal Component Analysis) 11 and LDA as feature extractors. Firstly, PCA is mathematically 
considered as an orthogonal linear transformation, which is a kind of a mapping function into other space. In pattern recognition fields, PCA is used for dimensionality reduction. In this paper, facial images $(40 \times 40)$ are represented as a $1 \times 1600$ vector. Since we collected many images per person, a face is considered as a vector of high dimension. Let the training set of $N$ face images be $\Gamma_{1}, \Gamma_{2}, \Gamma_{3}, \cdots, \Gamma_{N}$. The average face of the training set is

$$
\Psi=\frac{1}{N} \sum_{i=1}^{N} \Gamma_{i}
$$

Each face differs from the average by the vector

$$
\Phi_{i}=\Gamma_{i}-\Psi .
$$

For a $\Phi^{T}$ with zero empirical mean by Equation 2, the PCA transformation is given by

$$
Y=W^{T} \Phi=\Sigma V^{T}
$$

where $W^{T}$ is a transformation matrix and $W \Sigma V^{T}$ is the sigular value decomposition of $\Phi$.

We can consider $Y$ as a vector of a face in new space and recognize a person using this vector. Generally, PCA shows a good transformation and largely distributed vectors. However, it has a demerit if there are many data in same class. In this paper, we should find another method because we consider several posed faces in same persons.

LDA is a class specific method that it tries to shape the scatter in order to make it more reliable for classification. This method selects a transformation matrix, $W$ of Equation 3 in such a way that the ratio of the between-class scatter and the within-class scatter is maximized. Let the between-class scatter matrix be defined as

$$
\mathbf{S}_{\mathbf{B}}=\sum_{i=1}^{c} \mathbf{N}_{\mathbf{i}}\left(\mu_{i}-\mu\right)\left(\mu_{i}-\mu\right)^{T}
$$

and the within-class scatter matrix be defined as

$$
\mathbf{S}_{\mathbf{W}}=\sum_{i=1}^{c} \sum_{x_{k} \in X_{i}}\left(x_{k}-\mu_{i}\right)\left(x_{k}-\mu_{i}\right)^{T}
$$

where $\mu_{i}$ is the mean image of class $X_{i}$, and $N_{i}$ is the number of samples in class $X_{i}$. If $\mathbf{S}_{\mathbf{W}}$ is nonsingular, the optimal projection $W_{\text {opt }}$ is chosen as the matrix with orthonormal columns which maximizes the ratio of the determinant of the between-class scatter matrix of the projected samples to the determinant of the within-class scatter matrix of the projected samples, i.e.,

$$
W_{o p t}=\operatorname{argmax}_{W} \frac{\left|W^{T} S_{B} W\right|}{\left|W^{T} S_{W} W\right|}=\left[W_{1} W_{2} \cdots W_{m}\right]
$$


where $W_{i} \mid i=1,2, \cdots, m$ is the set of generalized eigenvectors of $\mathbf{S}_{\mathbf{B}}$ and $\mathbf{S}_{\mathbf{W}}$ corresponding to the $m$ largest generalized eigenvalues $\lambda_{i} \mid i=1,2, \cdots, m$, i.e.,

$$
\mathbf{S}_{\mathbf{b}} w_{i}=\lambda \mathbf{S}_{\mathbf{W}} w_{i}, \quad i=1,2, \cdots, m
$$

Note that there are at most $c-1$ nonzero generalized eigenvalues, and so an upper bound on $m$ is $c-1$, where $c$ is the number of classes. LDA has error rates lower than PCA and required less computation time[1]. When the number of training samples per class is small, it is known that PCA has better result than LDA [12. In this paper, we use more than 20 images per one class.

\section{Experimental Results and Analysis}

We performed several experiments to verify our approaches. Firstly, we collected 281 facial images. Secondly, we did preprocessing including histogram equalization and masking. Finally we recognized an input person by matching it with all trained faces. We can identify a person by selecting the index, which has the minimum error. In this experiment, we perform this process repeatedly. One of 7 trained persons was randomly inserted and the system recognized the detected facial image repeatedly.

\subsection{Collecting Facial Images}

For collecting facial images, wireless camera is used in order to enable to transfer video stream through radio frequency. This device is made up of wireless camera and $\mathrm{RF}$ receiver. The detailed information is explained in Table 2[16].

Table 2. Specification of wireless camera

\begin{tabular}{|c|c|}
\hline Image Sensor & $1 / 4$ " Color CCD \\
\hline Horizontal Resolution & 300 TV lines \\
\hline Sync & Internal \\
\hline PAL & 625 lines interlaced \\
\hline NTSC & 525 lines interlaced \\
\hline Light Sensitivity & 10 Lux \\
\hline Signal to Noise Ratio & $42 \mathrm{~dB}$ or more \\
\hline Gamma & 0.45 \\
\hline Frequency & $2.410 \mathrm{GHz}, 2.430 \mathrm{GHz}, 2.450 \mathrm{GHz}$ and $2.470 \mathrm{GHz}$ \\
\hline
\end{tabular}

In this paper, the robot moves around and collects facial images on the desk. It has tried to track a face and move a little step until it loses a face region. If it loses a face region, it returns a previous status and position to try it again. Server system carries the whole algorithm out and controls the robot by analyzed signal. Server system extracts facial images using OpenCV face detector and normalizes the size of faces into $40 \times 40$ (pixels) in every captured images. 


\subsection{Performance in Face Recognition}

We have performed experiments with captured images in real-time with our prototype system. We used a data set of 7 persons(more than 20 images per a person) for training and tried to recognize persons with newly captured images in indoor environment.

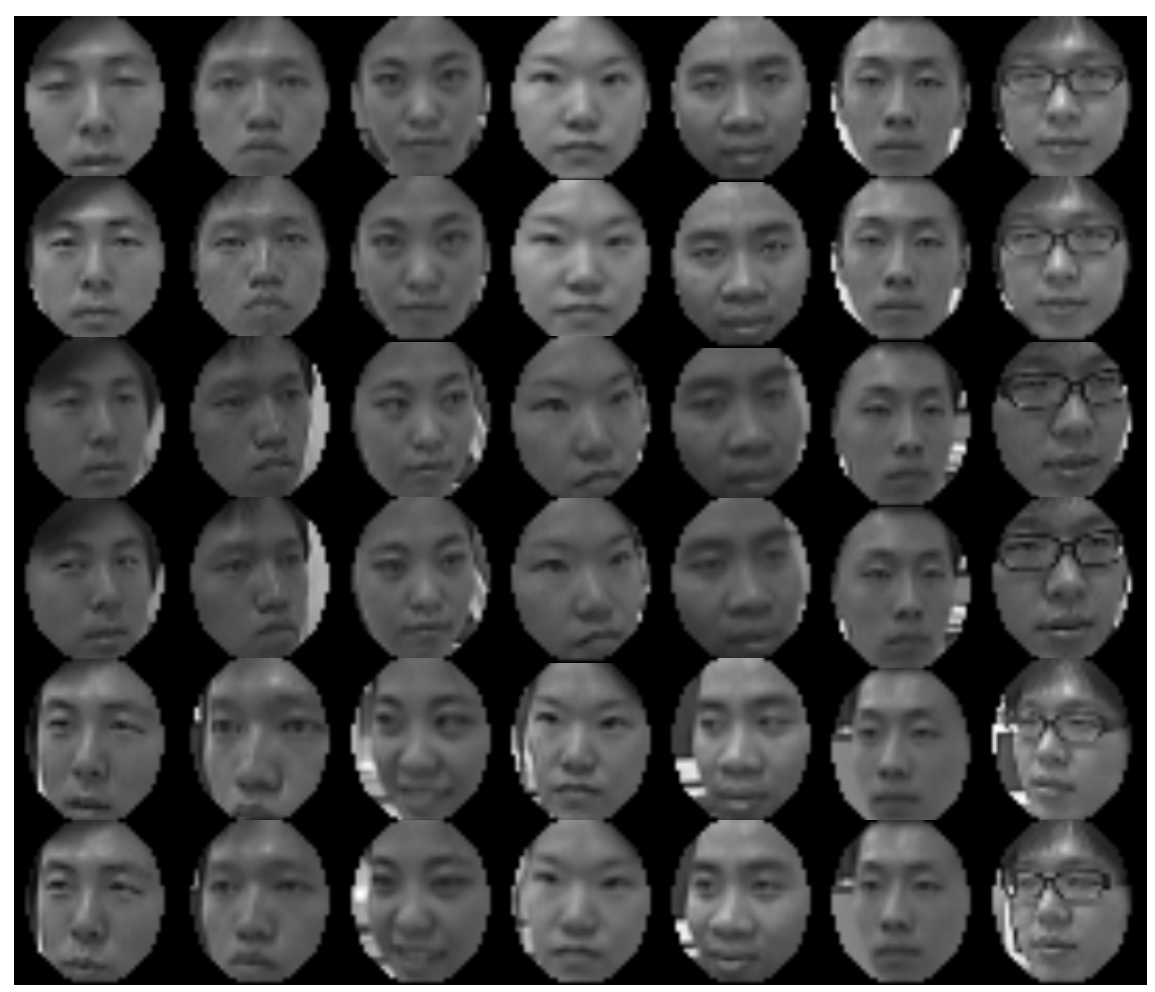

Fig. 3. Examples of collected facial images

This system can detect a face and recognize a person at 12-16 frame rates. This speed was measured from user-input to final stage with the result being dependent on the number of objects in an image. The system captures a frame from wireless camera through Bluetooth, preprocesses it, detects a face, extracts feature vectors and identifies a person. These whole stages have to be operated in real-time. In this system, movable robot always tries to train a manifold of each person. In a general way, even though training stage take much time, it is done in background process and user can not be aware of this process. We also measured the processing time of each stages using internal timer and the results were shown in Table 3 . Even though the training stage takes too much time to perform in real-time, we can turn the process into background running and we would make it as if it were 
Table 3. Speeds of each process

\begin{tabular}{|c|c|}
\hline Face detection & $111 \mathrm{~ms}$ \\
\hline Preprocessing(masking and histogram Equalization) & $67 \mathrm{~ms}$ \\
\hline PCA training & $250 \mathrm{~ms}$ \\
\hline LDA training & $221 \mathrm{~ms}$ \\
\hline Classification & $38 \mathrm{~ms}$ \\
\hline
\end{tabular}

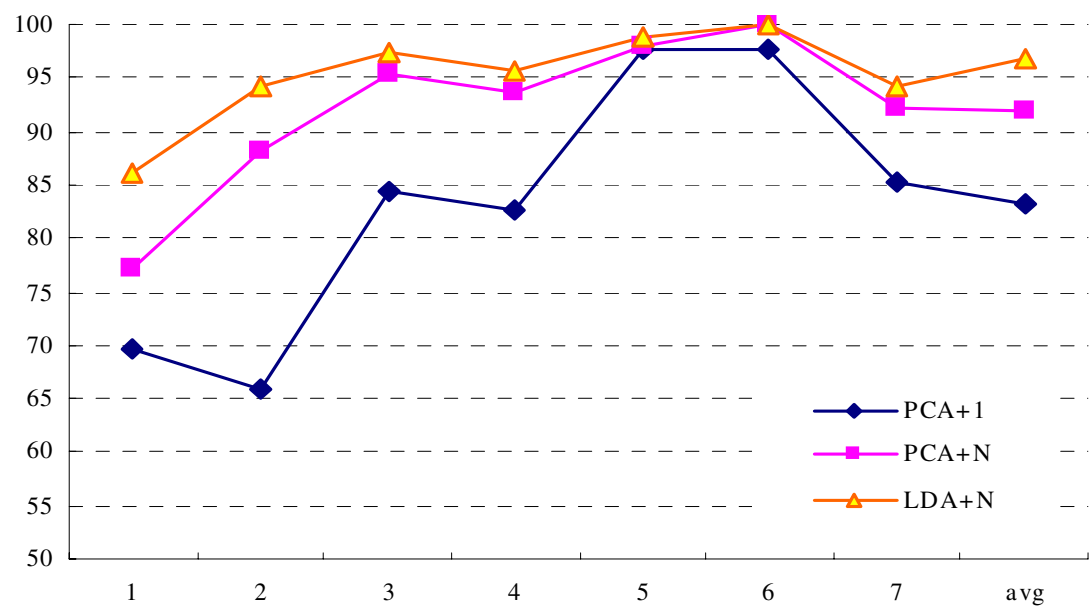

Fig. 4. Experimental results

done in real-time. We can also carry it out in real-time if the number of persons in training is small enough without other efforts.

The recognition performance of the system is highly dependent on accuracy of face extraction. In the next test, we measured the classification accuracy assuming correct face extraction, which means that we throw away the data wrongly extracted by OpenCV face detector.

This experiment was done by comparing three approaches in order to verify the effect of multiple training data. One is that we use the PCA method with only one image per person for training $(\mathrm{PCA}+1)$. Another is that we use the PCA method with $n$ images per person $(\mathrm{PCA}+\mathrm{N})$. The other is that we use the LDA method with more than $n$ images per person for training $(\mathrm{LDA}+\mathrm{N}) 11$ All of them use just an image for testing. The result in Fig. 4 shows that the LDA method with collected training images makes the result the best.

\section{Conclusions}

Until now, most of face recognition system have a fixed camera. Therefore they can not deal with various face pose. In order to cope with diverse facial images,

\footnotetext{
${ }^{1}$ In this paper, $\mathrm{N}=20$ was used.
} 
the face recognition system has to train many faces collected from well-designed studio or recognize a well-posed frontal face. In this paper, we construct a prototype system to throw these restricted conditions away.

In order to collect facial images enough in real environments, we organized several components such as a conventional PC, movable robot using LEGO Mindstorms NXT robot, and RF wireless communication. This system can actively capture an image sequence, find the features of face in the images, and recognize a person. In addition, it can collect the facial images of various poses due to movable robot, which enables this system to increase performance. We made several experiments and the result has confirmed us that the proposed system is better than conventional PC-based systems.

\section{Acknowledgments}

The author would like to thank Dr. Bum-Jae You at the Center for Cognitive Robotics, Korea Institute of Science and Technology and Dr. Sang-Woong Lee at Carnegie Mellon University for their kind supports and suggestions for this research.

\section{References}

1. Tan, X., Chen, S., Zhou, Z.-H., Zhang, F.: Face recognition from a single image per person: A survey. Pattern Recognition 39(9), 1725-1745 (2006)

2. Gilbert, J.M., Yang, W.: A Real-Time Face Recognition System Using Custom VLSI Hardware. In: Proc. of Computer Architectures for Machine Perceptron Workshop, New Orleans, USA, pp. 58-66 (1993)

3. Yang, F., Paindavoine, M., Abdi, H.: Parallel Implementation on DSPs of a Face Detection Algorithm. In: Proc. of International Conference on the Software Process, Chicago, USA (1998)

4. IBM ZISC036 Data Sheet: http://www.ibm.com

5. Zhou, S., Chellappa, R.: Rank constrained recognition under unknown illuminations. In: IEEE International Workshop on Analysis and Modeling of Faces and Gestures (2003)

6. Georghiades, A., Belhumeur, P., Kriegman, D.: From Few to Many: Illumination Cone Models for Face Recognition under Variable lighting and Pose. IEEE Transactions. on Pattern Analysis and Machine Intelligence 23(6), 643-660 (2001)

7. Gross, R., Matthews, I., Baker, S.: Appearance-Based Face Recognition and LightFields. IEEE Transactions on Pattern Analysis and Machine Intelligence 26(4), 449-465 (2004)

8. Blanz, V., Vetter, T.: Face Recognition Based on Fitting a 3D Morphable Model. IEEE Transactions on Pattern Analysis and Machine Intelligence 25(9), 1063-1074 (2003)

9. Sharad, S.: Introducing Embedded Design Concepts to Freshmen and Sophomore Engineering Students with LEGO MINDSTORMS NXT. In: IEEE International Conference on Microelectronic System Education, pp. 119-120. IEEE Computer Society Press, Los Alamitos (2007) 
10. LEGO Mindstorms NXT Hardware Developers Kit: http://mindstorms.lego.com/overview/nxtreme.aspx

11. Turk, M., Pentland, A.: Eigen faces for recognition. Journal of Cognitive Neuroscience 3, 71-86 (1991)

12. Martinez, A.M., Kak, A.C.: PCA versus LDA. IEEE Transaction on Pattern Analysis and Machine Intelligence 23(2), 228-233 (2001)

13. Viola, P., Jones, M.: Robust Real-time Face Detection. International Journal of Computer Vision 57(2) (2004)

14. http://sourceforge.net/projects/opencvlibrary/

15. http://mindstorms.lego.com/

16. http://www.miracleon.com/products/cm32c.php 